Erratum

\title{
Erratum to "A Network and Visual Quality Aware N-Screen Content Recommender System Using Joint Matrix Factorization"
}

\author{
Farman Ullah, Ghulam Sarwar, and Sungchang Lee \\ Department of Information \& Communication, Korea Aerospace University, Goyang 412-791, Republic of Korea \\ Correspondence should be addressed to Sungchang Lee; sclee@kau.ac.kr \\ Received 23 July 2014; Accepted 9 September 2014; Published 29 December 2014 \\ Copyright (C) 2014 Farman Ullah et al. This is an open access article distributed under the Creative Commons Attribution License, \\ which permits unrestricted use, distribution, and reproduction in any medium, provided the original work is properly cited.
}

In "A Network and Visual Quality Aware N-Screen Content Recommender System Using Joint Matrix Factorization," there was an error of the negative sign on the $x$-axis in Figures 9 and 10 . The right figures are provided as follows. 


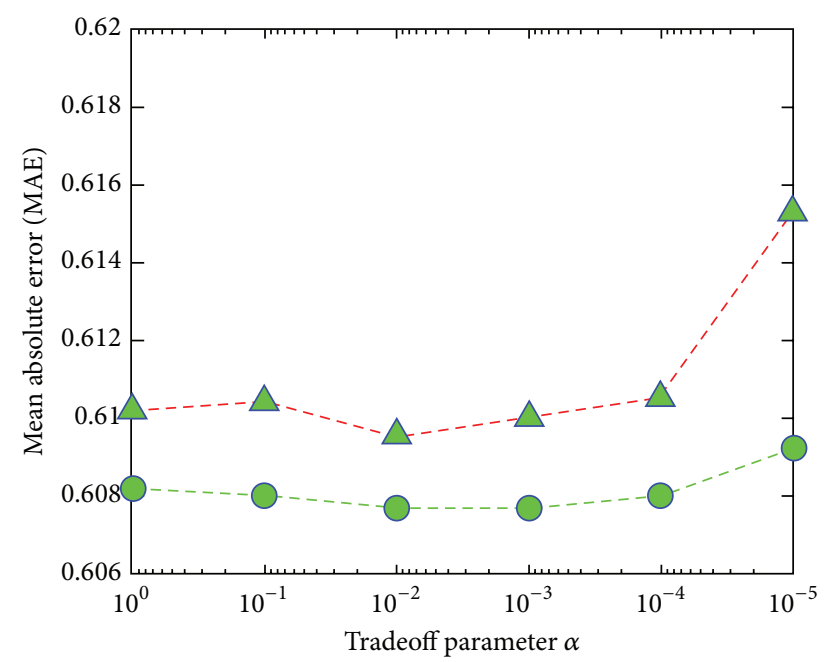

$-\triangle$ - Latent features dimensions (dimensionality number 10 )

- Latent features dimensions (dimensionality number 30)

FIGURE 9: Impact of tradeoff parameter $\alpha$ with different dimensions of latent features.

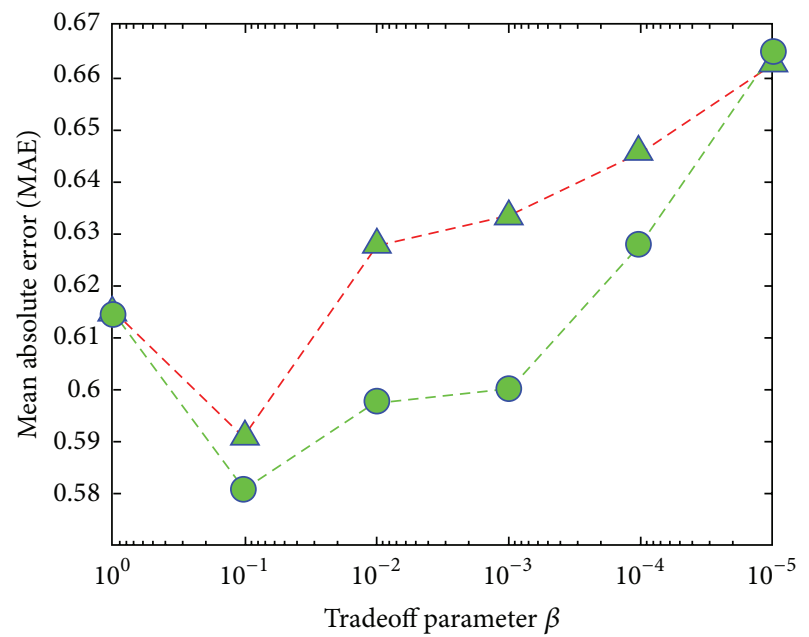

$-\triangle$ - Latent features dimensions (dimensionality number 10 )

- Latent features dimensions (dimensionality number 30 )

FIGURE 10: Impact of tradeoff parameter $\beta$ with different dimensions of latent features. 

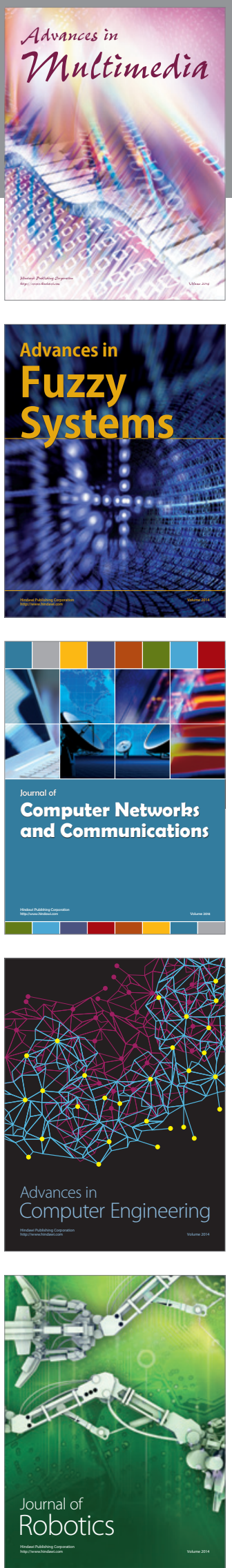

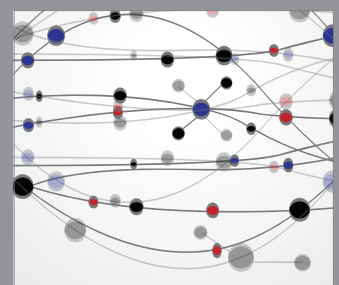

The Scientific World Journal
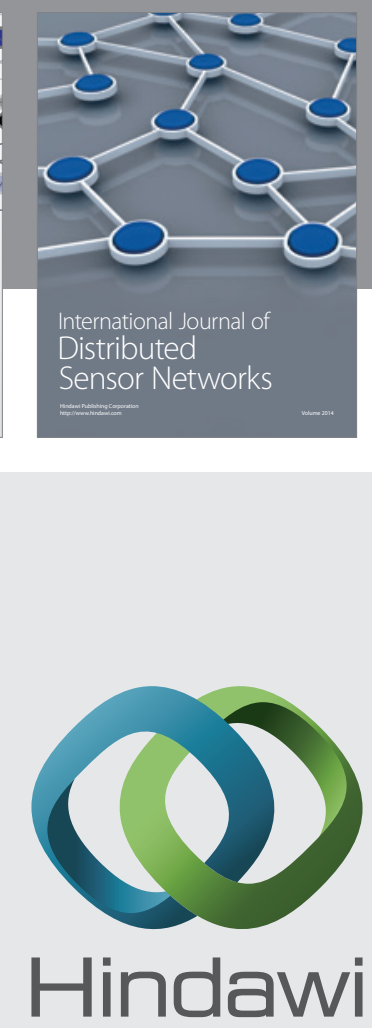

Submit your manuscripts at

http://www.hindawi.com
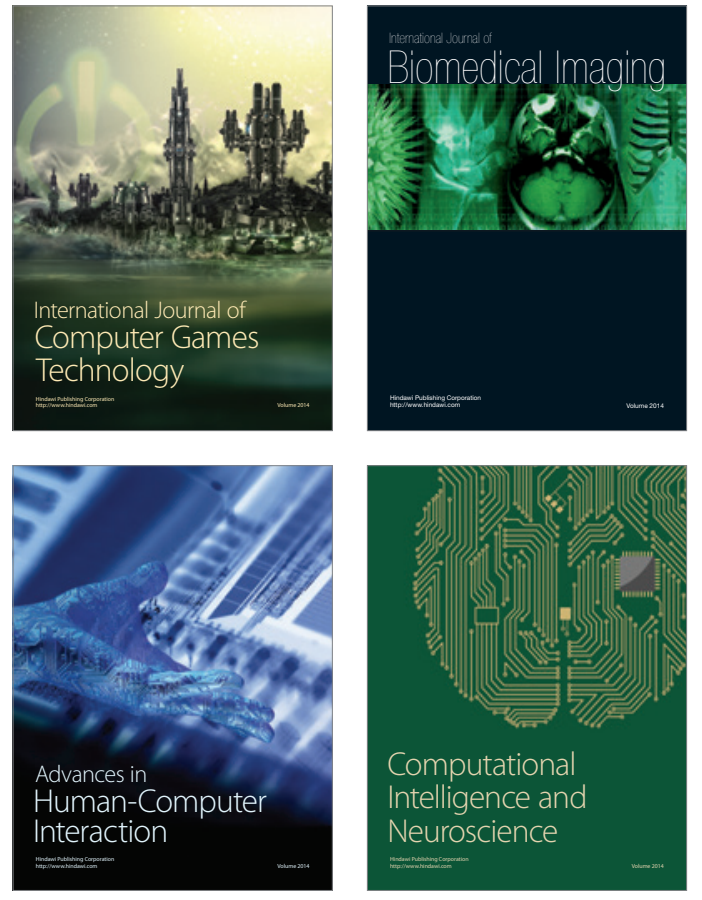
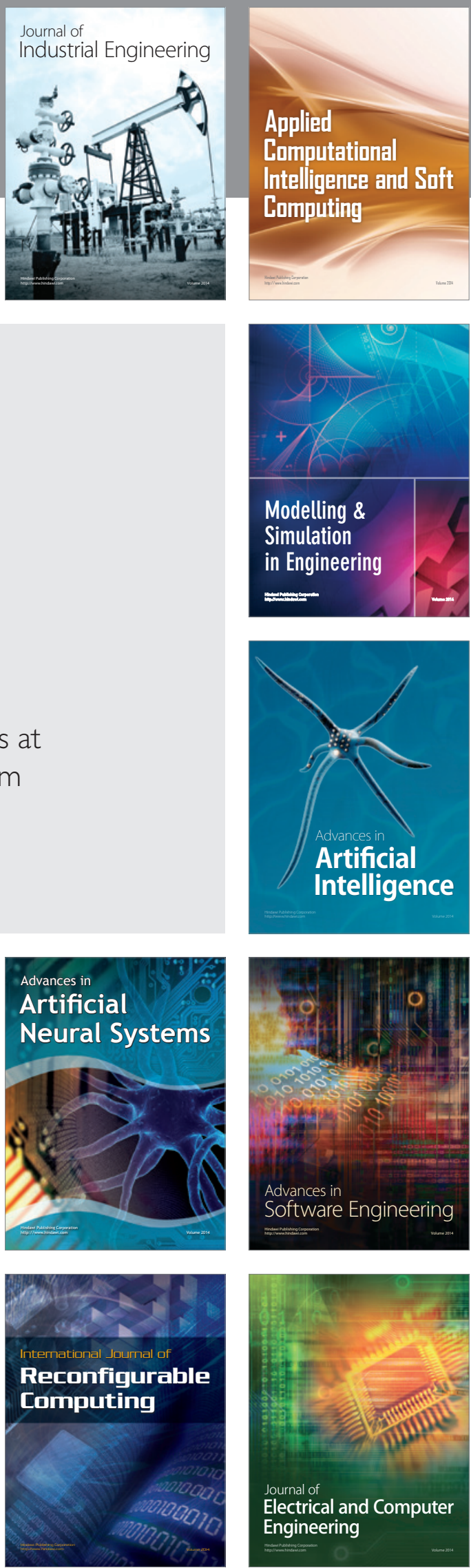